\section{Case Reports in Neurology}

\title{
A Case of Recurrent Ischemic Stroke due to Intravascular Lymphomatosis, Undiagnosed by Random Skin Biopsy and Brain Imaging
}

\author{
Yuichiro Ohya $^{a}$ Masato Osaki ${ }^{a}$ Shouta Sakai ${ }^{a}$ Shunsuke Kimura ${ }^{a}$ \\ Tatsuro Shimogamo ${ }^{b}$ Tetsuro Ago $^{c}$ Takanari Kitazono $^{c}$ \\ Shuji Arakawa ${ }^{a}$ \\ ${ }^{a}$ Department of Cerebrovascular Medicine, Stroke Center, Steel Memorial Yawata \\ Hospital, Kitakyushu, Japan; 'bepartment of Pathology, Steel Memorial Yawata Hospital, \\ Kitakyushu, Japan; ' ${ }^{C}$ epartment of Medicine and Clinical Science, Graduate School of \\ Medical Sciences, Kyushu University, Fukuoka, Japan
}

\section{Keywords}

Intravascular lymphoma · Random skin biopsy · Cryptogenic stroke

\begin{abstract}
Intravascular lymphoma (IVL) is a rare disease characterized by the proliferation of lymphoma cells in the lumen of the small blood vessels. Although early diagnosis of IVL is important to prolong survival of the patients, its atypical symptoms and clinical course often delay its diagnosis. More than half of the patients are diagnosed at autopsy. We report a 68-year-old man who presented with transient ideomotor apraxia and mildly elevated soluble interleukin2 receptor levels. He was initially diagnosed with aortogenic embolic stroke. He developed rapidly progressive neurological manifestations with enlargement of brain lesions on brain computed tomography and magnetic resonance imaging and died 3 months after symptom onset. The diagnosis of IVL could not be made by random skin biopsy, but was finally made at autopsy. For the early diagnosis, sufficient random skin biopsy or brain biopsy should be planned when suspected.




\section{Introduction}

Intravascular lymphoma (IVL) has an estimated annual incidence of 0.5 cases per $1,000,000$ [1]. It is classified as a systemic extranodal non-Hodgkin's lymphoma, characterized by selective growth of lymphoma cells within the lumen of small blood vessels [2]. The affinity of the lymphoma cells to the intravascular space is caused by the absence of CD29 and CD54 surface ligands, which may limit their ability to cross the vascular endothelium [3]. Although an early diagnosis may help prolong survival, it is often difficult to achieve because of an atypical presentation.

In the present study, we report a case of IVL initially presenting as cryptogenic stroke but followed by rapidly progressive neurological manifestations that remained unexplained until after the patient's death. This case highlights the need to be aware of IVL as a possible cause of rapidly progressive neurological manifestations, regardless of the initial symptoms.

\section{Case Presentation}

A 68-year-old man was admitted to our hospital because of a transient episode during which he had forgotten how to unlock a door. He had been treated for membranous glomerulonephritis and hypertension for almost 10 years and was also suspected to have idiopathic thrombocytopenic purpura.

Laboratory findings on admission showed thrombocytopenia, slight anemia, and impaired renal function, with the following values: leukocytes, 5,570/ $\mu \mathrm{L}$; erythrocytes, $365 \times$ $10^{4} / \mu \mathrm{L}$; hemoglobin, $10.6 \mathrm{~g} / \mathrm{dL}$; hematocrit, $32.7 \%$; platelets, $2.4 \times 10^{4} / \mu \mathrm{L}$; total protein, $5.2 \mathrm{~g} / \mathrm{dL}$; albumin, $3.1 \mathrm{~g} / \mathrm{dL}$; lactate dehydrogenase (LDH), $373 \mathrm{IU} / \mathrm{L}$; blood urea nitrogen, $29.9 \mathrm{mg} / \mathrm{dL}$; creatinine, $1.53 \mathrm{mg} / \mathrm{dL}$; C-reactive protein, $0.50 \mathrm{mg} / \mathrm{dL}$; D-dimer, $5.0 \mu \mathrm{g} / \mathrm{mL}$.

Brain magnetic resonance imaging (MRI) showed small lesions in the bilateral subcortical region of the frontal lobe, which were hyperintense on fluid-attenuated inversion recovery (Fig. 1a1) and diffusion-weighted (Fig. 1a2) imaging. Magnetic resonance angiography showed no stenosis of the carotid or intracranial arteries. We initiated aspirin treatment for suspected ischemic stroke.

Carotid ultrasonography, transthoracic echocardiography, 24-hour Holter electrocardiography, and computed tomography of whole body revealed no significant findings. Transesophageal echocardiography showed a 7-mm complicated lesion in the aortic arch, and the patient was diagnosed with aortogenic embolic stroke.

Additional laboratory tests showed elevated soluble interleukin-2 receptor (sIL-2R, $1,322 \mathrm{U} / \mathrm{mL}$ ) levels, which were considered to be a result of renal dysfunction, in the absence of fever or lymph node enlargement.

Three months after the onset of his symptoms, the patient's state of consciousness gradually worsened and he was admitted to our hospital after falling downstairs and hitting his head. His consciousness level was 12 points (E3, V4, M5) on the Glasgow Coma Scale. He presented left-half spatial neglect and left hemiplegia (Manual Muscle Test 4/5). Compared with the laboratory data at his first admission, his D-dimer $(17 \mu \mathrm{g} / \mathrm{mL}), \mathrm{LDH}(2,064 \mathrm{IU} / \mathrm{L})$, C-reactive protein $(5.7 \mathrm{mg} / \mathrm{dL})$, and sIL-2R $(1,462 \mathrm{U} / \mathrm{mL})$ levels were elevated.

Brain MRI at the second admission showed more and larger hyperintense lesions in the bilateral subcortical region on diffusion-weighted imaging (Fig. 1b). We suspected IVL on the basis of the elevated levels of LDH and sIL-2R, and the locations of the lesions in the brain. We conducted a lumbar puncture 3 days after the patient's second admission, and cerebro- 
spinal fluid analysis showed mild leukocytosis $(15 / \mu \mathrm{L})$ and elevated protein $(352 \mathrm{mg} / \mathrm{dL})$, but cytodiagnosis of the cerebrospinal fluid found no lymphoid tumor cells. We carried out a random skin biopsy 4 days after his second admission, which also failed to reveal any lymphoid tumor cells. The number and sizes of brain lesions had increased on brain MRI 10 days after the second admission (Fig. 1c). We planned to conduct a brain biopsy, but the patient's consciousness level worsened gradually and he died 11 days after his second admission.

We conducted an autopsy to determine the cause of death. His brain showed several edematous and soft lesions on macroscopic examination. We present here one demonstrable slice of the macroscopic examination and corresponding slice of the brain MRI. The brain had a discolored lesion on the subcortical region of the right parietal lobe (Fig. 2a, arrow). Findings of MRI on fluid-attenuated inversion recovery showed matching (arrow) and mismatching (arrowhead) with the macroscopic findings (Fig. 2b). The mismatched hyperintense signal on MRI in the subcortical region of the left parietal lobe might be an ischemic edematous change due to occlusion of microvasculature, but it was not evident in the macroscopic observation of the brain.

Microscopic examination revealed lymphoid tumor cells confined to the small vessels in the brain and other organs, including the spleen, bone marrow, kidney, and liver. The lymphoid tumor cells demonstrated immunoreactivity for the pan-B cell marker CD20 (Fig. 3). Autopsy thus determined a final diagnosis of death due to intravascular large B-cell lymphoma.

\section{Discussion}

IVL is known to be difficult to diagnose and is only diagnosed postmortem in $>50 \%$ of cases because of the lack of specific diagnostic procedures, including specific symptoms and MRI findings [4, 5]. However, if an early diagnosis is made, remission may be achieved by prompt administration of systemic chemotherapy [4]. Fonkem et al. [5] compared the survival of patients diagnosed postmortem versus premortem and found that a premortem diagnosis was associated with significantly longer survival (17.0 months, range 0.5-71.1 months, $n=98$, vs. 3.5 months, range $0.1-30.0$ months, $n=150 ; p=0.0001$ ). An early diagnosis is thus an important factor in prolonging survival.

Two main types of IVL, Asian and Western variants, have been identified according to their geographical origins and variable clinical presentations [6]. The brain and skin are the most commonly affected organs in the Western variant, while the bone marrow, spleen, and liver are the most common ones in the Asian variant. The latter type may also include hemophagocytic syndrome, which presents as pancytopenia and hepatosplenomegaly. However, it is difficult to make a clear distinction between these two types of IVL because their symptoms often coexist. Fonkem et al. [1] reported that most cases showed neurological symptoms, commonly including cognitive impairment/dementia, paralysis, and seizures. However, these symptoms are not specific and are therefore difficult to attribute to IVL.

Yamamoto et al. [7] reported that MRI findings of IVL could be categorized as follows: (1) infarct-like lesions; (2) nonspecific white matter lesions; (3) meningeal enhancement; (4) mass-like lesions; (5) hyperintense lesions in the pons on T2WI. These different MRI patterns could be explained by variations in the affected vessels. However, because these findings do not exist simultaneously and no individual finding is specific, IVL is difficult to diagnose, even by MRI. 
Laboratory findings can lead to a suspicion of malignant lymphoma, with anemia being the most frequent cytopenia (63\%), while leukopenia (24\%) and thrombocytopenia (29\%) do not usually occur without anemia. Elevated serum LDH and $\beta 2$-microglobulin levels are observed in $>80 \%$ of patients [8]. Elevated sIL-2R levels are relatively specific to malignant lymphoma, though mildly elevated levels may also be associated with renal dysfunction, autoimmune disease, or other malignant tumors. In the present case, we considered renal dysfunction to be the likely cause of the patient's elevated sIL-2R levels because of the absence of fever or lymph node enlargement. Tsujioka et al. [9] reported a median serum sIL$2 \mathrm{R}$ level in patients with malignant lymphoma of $1,330 \mathrm{U} / \mathrm{mL}$.

IVLs are often diagnosed by bone marrow, liver, or skin biopsy, and a previous report indicated that it could also be diagnosed by biopsy of the gastrointestinal tract [8]. Fluorodeoxyglucose-positron emission tomography/computed tomography may help in selecting a biopsy target [10]. We selected a random skin biopsy for initial pathological examination in the present case, because this test is relatively noninvasive and highly sensitive. Matsue et al. [11] reported that random skin biopsies were positive in 10 of 12 patients with IVL (83.3\%), suggesting that this technique is highly sensitive for the diagnosis of IVL. However, Maekawa et al. [12] reported that adipose tissue contained a higher percentage of atypical lymphoid cells than other layers of the skin. The negative skin biopsy result in the current case may have been due to the fact that the biopsy section did not include sufficient adipose tissue, suggesting that the depth of the biopsy was insufficient. Brain biopsy should thus have been performed without delay when no tumor cells were detected by skin biopsy and if the patient's condition was suitable. It is also possible that other biopsy targets could have been investigated following the negative skin biopsy, including the gastrointestinal tract, which could be easily biopsied.

\section{Conclusion}

Its rarity and atypical presentation make IVL difficult to diagnose. Laboratory findings may raise suspicion of malignant lymphoma, and suspected IVL should be investigated further by fluorodeoxyglucose-positron emission tomography/computed tomography or biopsy of skin, bone marrow, or gastrointestinal tract, which are relatively noninvasive and easy procedures. If skin biopsy is performed, it is essential to ensure that the sample includes sufficient adipose tissue.

\section{Statement of Ethics}

Because this is a retrospective case report, ethical approval was waived. However, informed consent was obtained from the patient to publish his case in accordance with the Declaration of Helsinki.

\section{Disclosure Statement}

All authors declare no disclosures. 
Ohya et al.: A Case of Recurrent Ischemic Stroke due to Intravascular Lymphomatosis, Undiagnosed by Random Skin Biopsy and Brain Imaging

\section{Author Contributions}

Yuichiro Ohya reviewed clinical data, made literature search, and drafted the manuscript. Masato Osaki, Shouta Sakai, Shunsuke Kimura, Tatsuro Shimogamo, Tetsuro Ago, Takanari Kitazono, and Shuji Arakawa revised the manuscript.

\section{References}

1 Fonkem E, Dayawansa S, Stroberg E, Lok E, Bricker PC, Kirmani B, Wong ET, Huang JH: Neurological presentations of intravascular lymphoma (IVL): meta-analysis of 654 patients. BMC Neurol 2016;16:9. Ponzoni M, Ferreri AJ, Campo E, Facchetti F, Mazzucchelli L, Yoshino T, Murase T, Pileri SA, Doglioni C, Zucca E, Cavalli F, Nakamura S: Definition, diagnosis, and management of intravascular large B-cell lymphoma: proposals and perspectives from an international consensus meeting. J Clin Oncol 2007;25:3168-3173.

-3 Ponzoni M, Arrigoni G, Gould VE, Del Curto B, Maggioni M, Scapinello A, Paolino S, Cassisa A, Patriarca C: Lack of CD 29 (beta1 integrin) and CD 54 (ICAM-1) adhesion molecules in intravascular lymphomatosis. Hum Pathol 2000;31:220-226.

-4 Hundsberger T, Cogliatti S, Kleger GR, Fretz C, Gähler A, Anliker M, Fournier JY, von Moos R, Tettenborn B, Driessen C: Intravascular lymphoma mimicking cerebral stroke: report of two cases. Case Rep Neurol 2011;3:278-283.

-5 Fonkem E, Lok E, Robinson D, Gautam S, Wong ET: The natural history of intravascular lymphomatosis Cancer Med 2014;3:1010-1024.

-6 Ferreri AJ, Campo E, Seymour JF, Willemze R, Ilariucci F, Ambrosetti A, Zucca E, Rossi G, LópezGuillermo A, Pavlovsky MA, Geerts ML, Candoni A, Lestani M, Asioli S, Milani M, Piris MA, Pileri S, Facchetti F, Cavalli F, Ponzoni M: Intravascular lymphoma: clinical presentation, natural history, management and prognostic factors in a series of 38 cases, with special emphasis on the 'cutaneous variant'. Br J Haematol 2004;127:173-183.

-7 Yamamoto A, Kikuchi Y, Homma K, O'uchi T, Furui S: Characteristics of intravascular large B-cell lymphoma on cerebral MR imaging. AJNR Am J Neuroradiol 2012;33:292-296.

8 Ponzoni M, Ferreri AJ: Intravascular lymphoma: a neoplasm of homeless lymphoma. Hematol Oncol 2006;24:105-112.

9 Tsujioka T, Kishimoto M, Kondo T, Matsuoka A, Tasaka T, Sugihara T, Wada H, Tohyama K: The impact of serum soluble interleuikin-2 receptor levels on the diagnosis of malignant lymphoma. Kawasaki Medical Journal 2011;37:19-27.

10 Boslooper K, Dijkhuizen D, van der Velden AW, Dal M, Meilof JF, Hoogenberg K: Intravascular lymphoma as an unusual cause of multifocal cerebral infarctions discovered on FDG-PET/CT. Neth J Med 2010;68:261-264.

-11 Matsue K, Asada N, Odawara J, Aoki T, Kimura S, Iwama K, Fujiwara H, Yamakura M, Takeuchi M: Random skin biopsy and bone marrow biopsy for diagnosis of intravascular large B cell lymphoma. Ann Hematol 2011;90:417-421.

12 Maekawa T, Komine M, Murata S, Fukushima N, Ohtsuki M: Random skin biopsy of patients with intravascular large B-cell lymphoma associated with thrombocytopenia and coagulation abnormalities: proposal of a modified biopsy method. J Dermatol 2015;42:318-321. 


\section{Case Reports in Neurology}
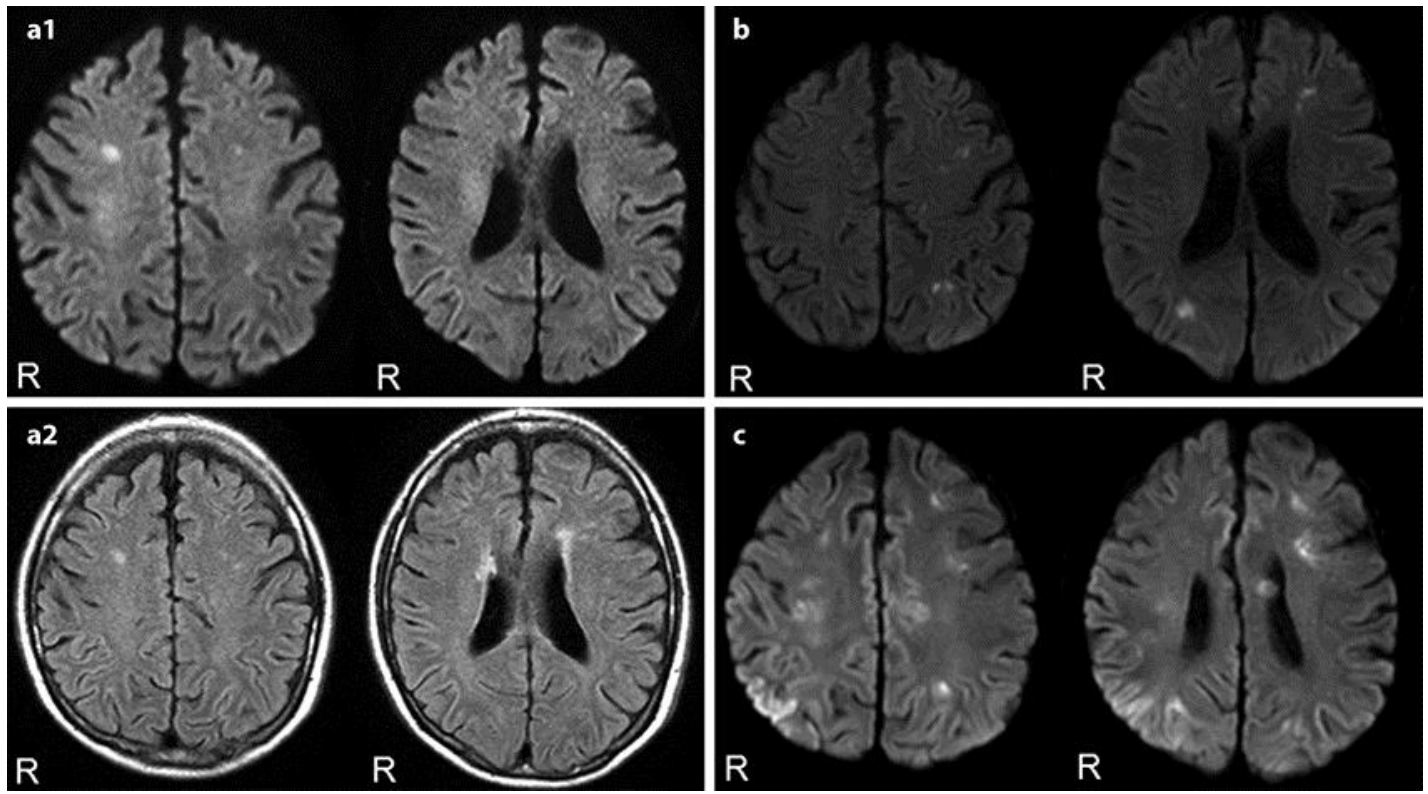

Fig. 1. Diffusion-weighted imaging (DWI; a1) and fluid-attenuated inversion recovery (a2) showed minimal hyperintense lesions in the bilateral subcortical region of the frontal lobe. $\mathbf{b}$ DWI showed more and larger hyperintense lesions in the bilateral subcortical region on the second admission. c DWI showed increased number and sizes of lesions 10 days after the second admission. $\mathrm{R}$, right side.
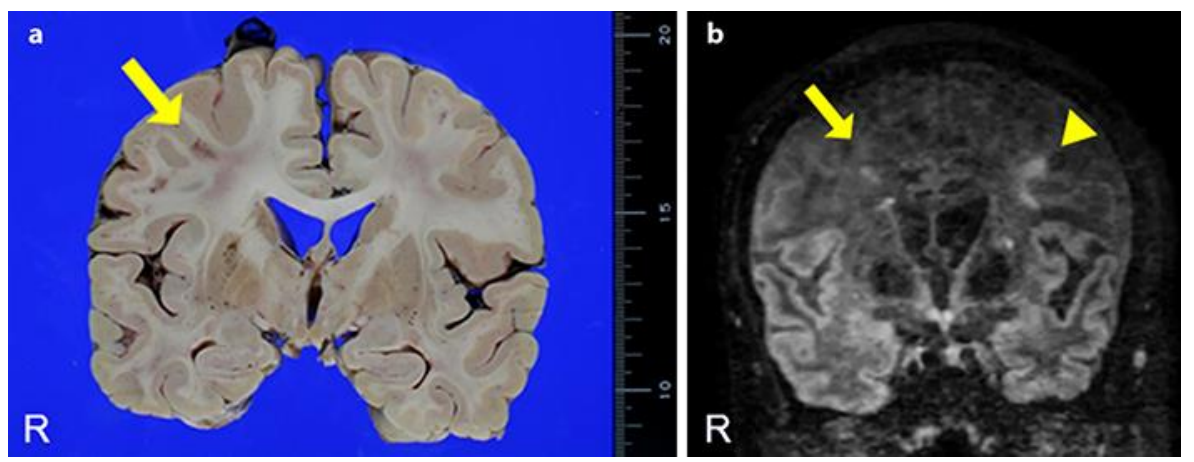

Fig. 2. a The brain had a discolored lesion on the subcortical region of the right parietal lobe (arrow). b Fluid-attenuated inversion recovery at coronal plane showed hyperintense lesions (arrow and arrowhead) in the bilateral subcortical region 10 days after the second admission. $\mathrm{R}$, right side. 


\section{Case Reports in Neurology}
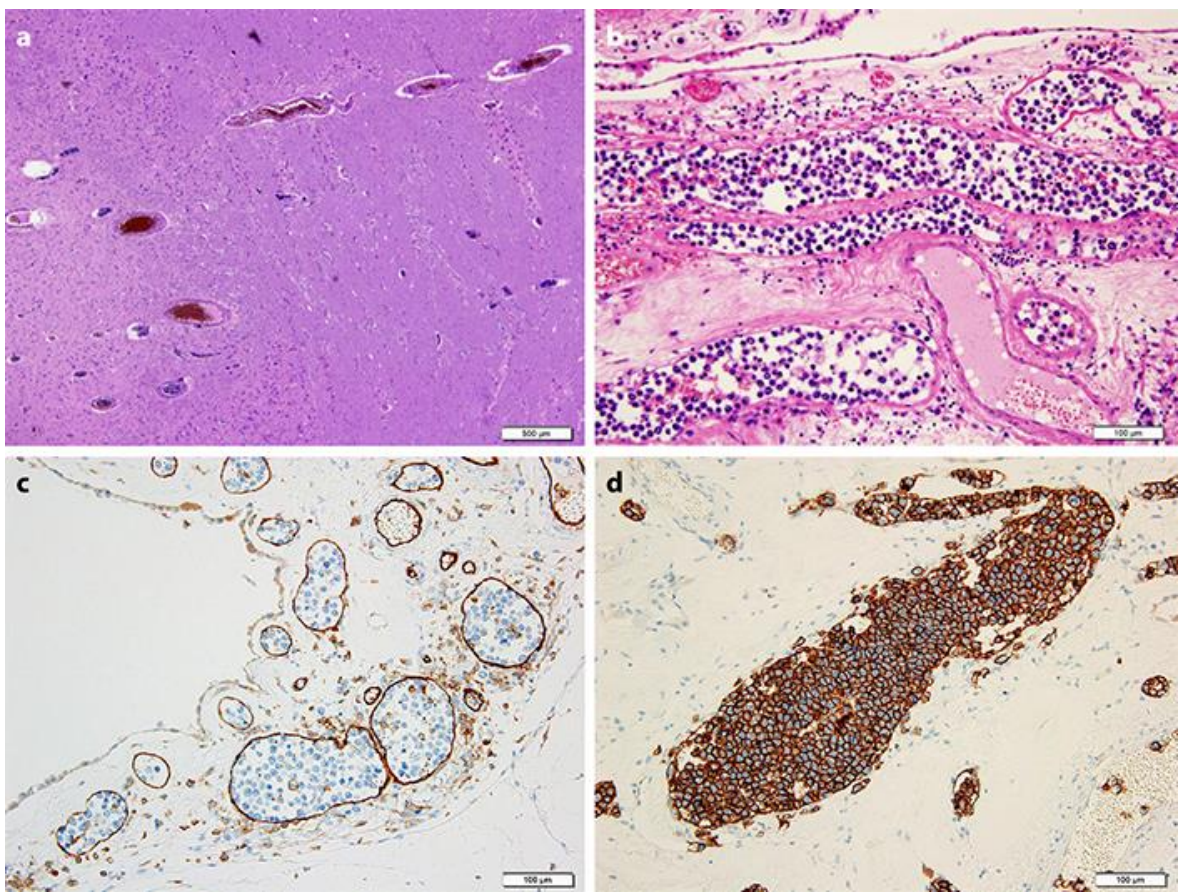

Fig. 3. a Lymphoid cells located in the small vessels of the subcortical region of the parietal lobe (hematoxylin-eosin staining, original magnification $\times 40$ ). b Lymphoid cells located in the small vessels of the choroid (hematoxylin-eosin staining, original magnification $\times 200$ ). c CD31 staining showed intravascular location of lymphoid cells (CD31 immunostaining, original magnification $\times 200$ ). $\mathbf{d}$ Lymphoid cells positive for CD20 staining located in the small vessels (CD20 immunostaining, original magnification $\times 200$ ). 